\title{
DEMONSTRATION OF TERRASAR-X SCANSAR PERSISTENT SCATTERER INTERFEROMETRY
}

\author{
Fernando Rodriguez Gonzalez ${ }^{(1)}$, Ramon Brcic ${ }^{(1)}$, Nestor Yague-Martinez ${ }^{(1,2)}$, \\ Robert Shau $^{(1)}$, Alessandro Parizzi ${ }^{(1)}$, Nico Adam ${ }^{(1)}$ \\ ${ }^{(1)}$ Remote Sensing Technology Institute (IMF), German Aerospace Center (DLR) \\ Oberpfaffenhofen,82234 Weßling, Germany.Email: Fernando.RodriguezGonzalez@dlr.de \\ (2) Chair of Remote Sensing Technology, Technical University Munich (TUM) \\ Arcisstraße 21, 80333 Munich, Germany
}

\begin{abstract}
The concept of PSI Wide Area Product was introduced in ESA's GMES Terrafirma project [1] in preparation for the upcoming Sentinel-1 mission. Sentinel-1 provides unprecedented capabilities for wide area mapping due to the coverage of the IW (Interferometric Wide swath) mode, which is the predefined mode over land. The extension of the PSI technique to wide area deformation mapping presents many technical challenges, especially in non-urban areas and in mountainous areas [2]. The Integrated Wide Area Processor (IWAP) [3] has been developed at DLR for this purpose. This paper presents the processor extensions performed in order to support the ScanSAR and TOPS SAR acquisition modes in preparation for the Sentinel-1 mission.
\end{abstract}

\section{INTRODUCTION}

The Sentinel-1 system has been conceived in order to provide wide swath SAR interferometric capabilities. A wide swath is achieved by means of the novel TOPS (Terrain Observation by Progressive Scan) SAR acquisition mode [4]. In this mode the radar beam cyclically scans a group of neighbouring swaths, as it is also performed in the ScanSAR acquisition mode. Furthermore, in TOPS the antenna is systematically steered forwards during the scan of a burst, eliminating thus the main drawbacks of ScanSAR, namely the effects of scalloping and the azimuth variant noise equivalent $\sigma_{0}$ and signal-to-ambiguity ratio.

Nevertheless, the spectral characteristics of the focussed SAR complex images (linear Doppler Centroid variation over azimuth) and the interferometric processing techniques are analogous for ScanSAR and TOPS SAR [5][6]. The main difference is the higher azimuth coregistration accuracy required for TOPS SAR, which is due to its higher Doppler Centroid variations within a burst caused by the antenna forward steering.

In the framework of ESA GMES Terrafirma project (ESRIN/Contract no. 4000109669/13/I-AM) as well as in order to support ESA in its Sentinel-1A Commissioning Phase activities (ESA Contract No. 4000111074/14/NL/MP/lf) [7], DLR's IWAP processor has been extended to support both burst acquisition modes: ScanSAR and TOPS SAR.

During the Terrafirma project there were no Sentinel-1 stacks available. As a consequence, the processing demonstration has been performed with a stack of 65 TerraSAR-X ScanSAR images. It covers an area of approximately 100x100 km surrounding Lake Mead, an artificial water reservoir impounded by the Hoover Dam. The scene is characterised by high topography variations, from 200 to 2400 meters. The stack was acquired from August 2008 to January 2011 and its master on the $10^{\text {th }}$ December 2009. During this period, the water elevation of Lake Mead progressively descends from approximately $337.5 \mathrm{~m}$ to $332.5 \mathrm{~m}$ [8] (see Fig. 1-left). Seasonal water elevation changes can be also clearly seen. The time baseline distribution of the stack is presented in Fig. 1-right.

\section{METHODOLOGY}

\section{InSAR Processing}

The principles of the implemented burst mode interferometric processing have been presented in [6]. The co-registration approach is DEM based geometric co-registration with correction of a residual offset (both in range and in azimuth) per interferogram determined either by Incoherent Cross Correlation (ICC) or by Enhanced Spectral Diversity (ESD) [5]. In this test case the offset has been determined by ICC, while ESD has been used for quality control.

Selected interferograms are depicted in Fig. 2-left and center in order to illustrate the atmospheric conditions within the stack. High turbulence and stratification can be observed. The acquisitions have been performed in the late afternoon (ascending orbit), when atmospheric turbulence is higher. The high atmospheric stratification effect is due to the big height variations within the scene, causing approximately two fringes in the example shown.

\section{PSI Processing}

The approach designed for PSI processing is conditioned by the elongated shape of the bursts, which have an azimuth length on the order of only 

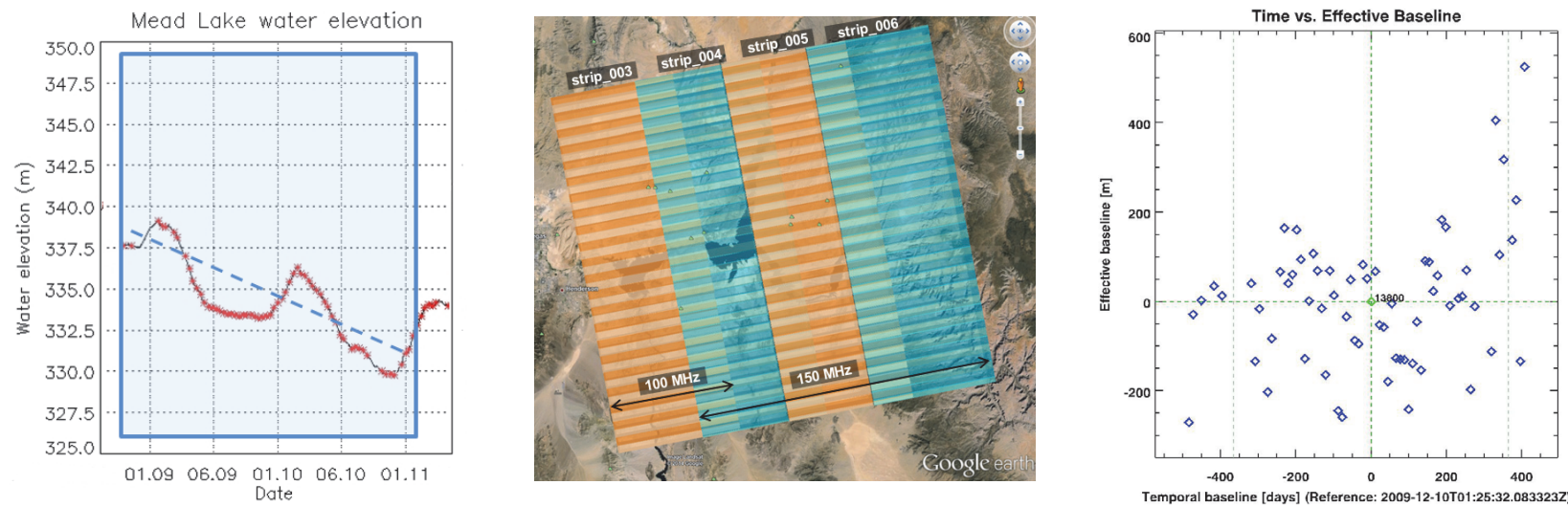

Figure 1. TerraSAR-X ScanSAR Stack: (left) Lake Mead water level variation during the acquisition time span (source [8]) with acquisitions depicted as red dots and time span in blue; (center) beams and burst of ScanSAR acquisitions (Image Landsat () 2014 Google); (right) time baseline distribution.
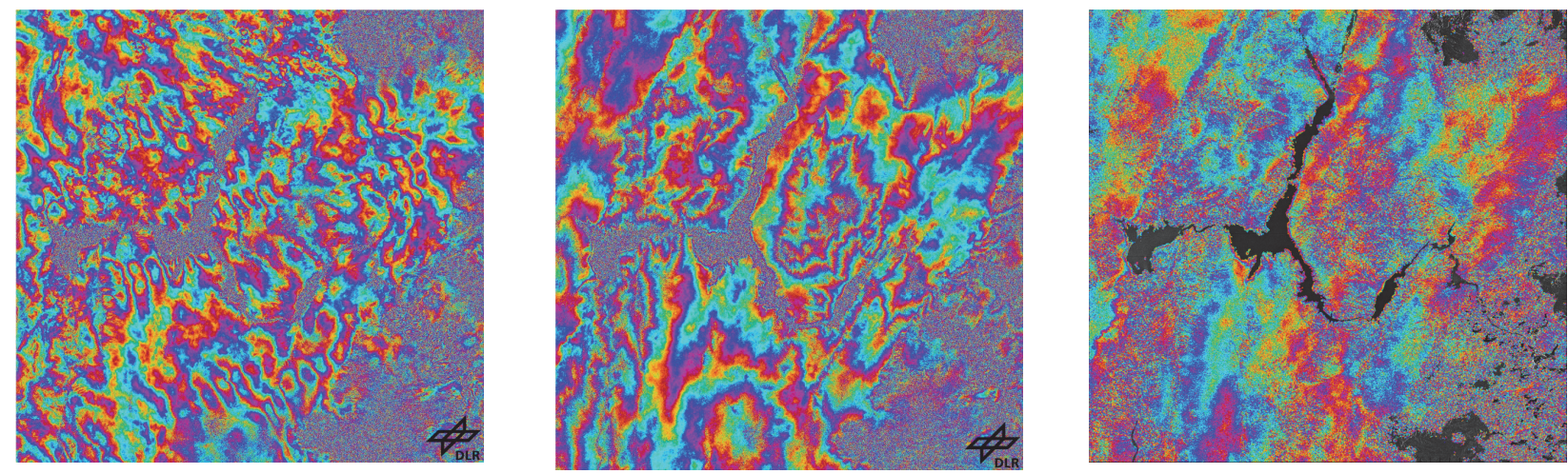

Figure 2. (Left) differential interferogram slave 2009-01-25; (center) differential interferogram slave 2009-07-09; (right) differential PS phases for interferogram slave 2009-07-09 after stratification mitigation.

$2.3 \mathrm{~km}$. Strategies based on burst per burst processing and mosaicking of burst level PSI estimates are not feasible. Our approach is to process all bursts jointly.

In order to perform PSI detection the scene is partitioned into non-overlapping areas, each corresponding to the central part of the bursts. PSs are detected within each of this burst areas according to an SCR threshold and sub-pixel position determination. Once detected, PSs are handled independently of their origin. Arcs are generated from burst to burst, including beam to beam. Thus it is useful to review the impact of the Doppler Centroid in the arc double differences. The SAR impulse response function in zero Doppler geometry in the case of an observation squint angle $\beta$ is given by [9]

$$
\begin{aligned}
\mathrm{s}(\tau, t)= & C \cdot s_{r}\left(\tau-\tau_{0}\right) \cdot s_{a}\left(t-t_{0}-\frac{c}{2} \cdot \frac{\tan \beta}{v} \cdot\left(\tau-\tau_{0}\right)\right) \\
& \cdot \exp \left(-j \frac{4 \pi}{\lambda} r_{0}\right) \cdot \exp \left(j 2 \pi f_{D C}\left(t-t_{0}\right)\right) \\
& \cdot \exp \left(-j 2 \pi f_{0}(1-\cos \beta)\left(\tau-\tau_{0}\right)\right)
\end{aligned}
$$

where $\tau, t$ are the fast and slow time, $\tau_{0}, t_{0}, r_{0}$ are the closest approach times and distance, $C$ is a complex constant, $s_{r}$ and $s_{a}$ the compressed range and azimuth envelopes, $v$ effective platform forward velocity, $f_{0}$ the radar frequency, $f_{D C}$ the Doppler Centroid and $\lambda$ the wavelength. In the presence of a small squint the last exponential term can be neglected. According to this, the phase of a PS 1 in the master scene extracted with an azimuth subpixel error $\Delta t_{P T A, 1}$ is given (up to a constant) by

$$
\phi_{1}^{M}=-\frac{4 \pi}{\lambda} r_{01}{ }^{M}+2 \pi f_{D C, 1}^{M} \Delta t_{P T A, 1}
$$

and the phase of the same point extracted from a slave image with an azimuth co-registration error of $\Delta t_{C O}$ is given by

$$
\phi_{1}^{S}=-\frac{4 \pi}{\lambda} r_{01}^{S}+2 \pi f_{D C, 1}^{S}\left(\Delta t_{P T A, 1}-\Delta t_{C O}\right)
$$

where the super-index $M$ and $S$ denote master and slave images. Let $\phi_{i}^{I}=\phi_{i}^{M}-\phi_{i}^{S}$ be the interferometric phase of a point $i$. Then the phase of a double difference between points 1 and 2 is given by

$$
\begin{aligned}
\phi_{1}^{I}-\phi_{2}^{I}= & -\frac{4 \pi}{\lambda}\left(\Delta r_{0,1}^{M \rightarrow S}-\Delta r_{0,2}^{M \rightarrow S}\right) \\
& +2 \pi \Delta f_{D C, 1}^{M \rightarrow S} \Delta t_{P T A, 1}-2 \pi \Delta f_{D C, 2}^{M \rightarrow S} \Delta t_{P T A, 2} \\
& +2 \pi\left(f_{D C, 1}^{S}-f_{D C, 2}^{S}\right) \Delta t_{C O}
\end{aligned}
$$



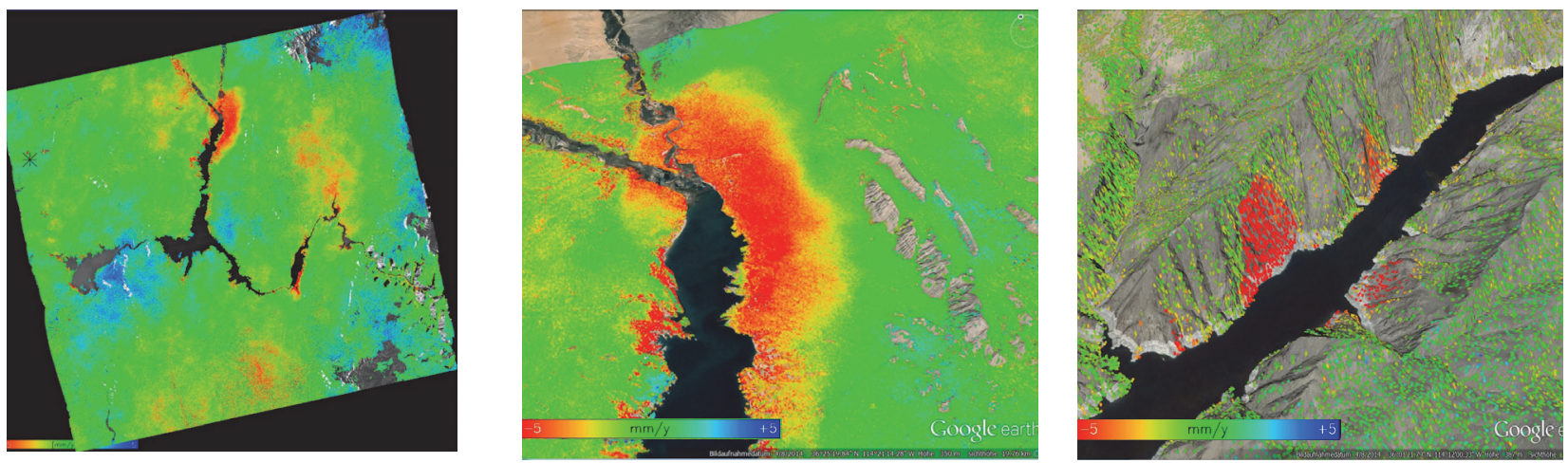

Figure 3. Mean linear deformation rate estimates: (left) overview; (center) oval subsidence pattern in northern area; (right) landslide. $3 D$ visualizations (center and right) generated with Google Earth.
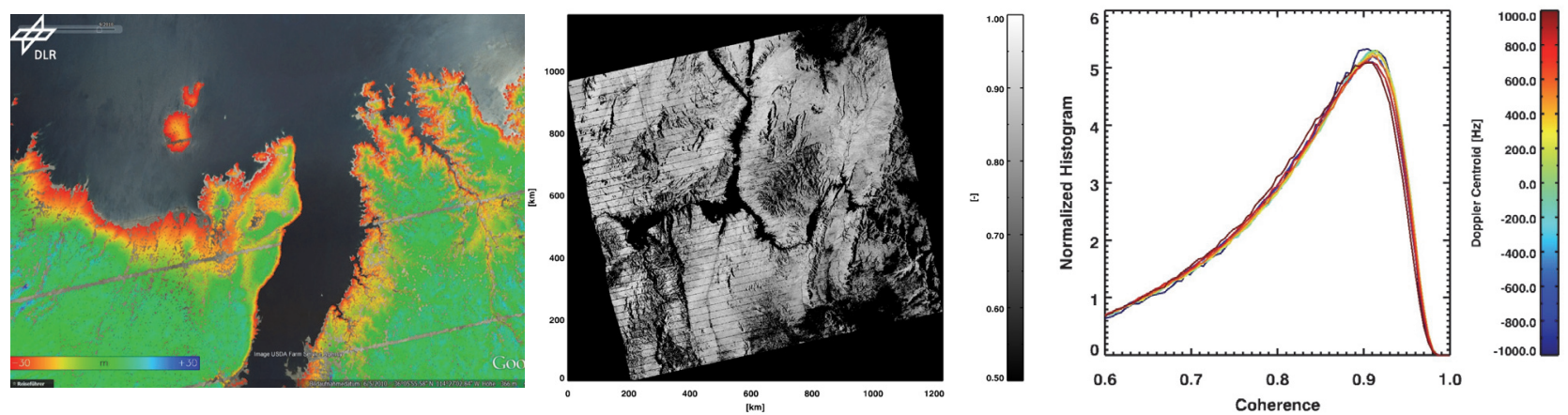

Figure 4. (Left) Topographic update estimates (visualization: Image USDA Farm Service Agency, Google Earth); (center) local average coherence in 100x100 grid (minimum 10 PS per cell); (right) histograms of coherence for different Doppler Centroid values (200 Hz bins, with the histogram color coded with center Doppler Centroid).

where the notation $\Delta[]_{i}^{M \rightarrow S}=[]_{i}^{M}-[]_{i}^{S}$ denotes the difference between master and slave acquisitions for the point $i$. The first term is the one we exploit for inverting difference of deformation and residual height. The second term is due to the subpixel position determination errors. Nevertheless its proportionality factors $\Delta f_{D C, i}^{M \rightarrow S}$ are very small for current SAR missions due to accurate burst synchronization and attitude control. By performing some prior oversampling and/or point target analysis this term can be minimized. The third term is much more important, since it depends on the difference of Doppler Centroids between points 1 and 2, which for ScanSAR and TOPS SAR can be very high (especially in the case of arcs between different bursts). This term is minimised by means of an accurate co-registration in the stacking process. Thus, as long as the co-registration has been done accurately, it is possible to use any standard PSI estimator.

Due to the high height variation in this test site it is required to mitigate the stratified atmospheric delay in order to i) avoid biased deformation components correlated with height and to ii) generate reliable arcs between different heights for a robust reference network construction and inversion. The approach used here has been iterative: based on an initial PS processing, the unwrapped PS phases have been used to fit a linear height-to-phase model in the mountain located in the central area and its surroundings. Note that this data based approach has the risk of compensating deformation components which are correlated with height. Weather model data based approaches would avoid this risk.

The further PSI processing has been performed using standard techniques described in [2][3].

\section{RESULTS}

An overview of the deformation estimates is provided in Fig.3-left. A spatial ramp has been compensated, reducing thus low pass residual components. Nevertheless, still residual low pass atmospheric signals can be seen in the results. This may be due to the high atmospheric turbulence as well as to residuals from the stratification mitigation and to the time span of the stack.

Further from these effects, we observe a big subsidence pattern in the northern area of Lake Mead (Fig.3center). In [10] it was also observed that this area did not follow the global deformation pattern. The explanation that the authors provided [10] was that the local pattern possibly was caused by the poroelastic response of a sedimentary layer with groundwater communicated with the lake. The authors explain that a 
high lake level may induce a poroelastic uplift of the surface of the sedimentary layer. Since in our acquisition time span the water level goes down, we observe subsidence, which would be consistent with the explanation in [10].

A subsidence which a priori seems a landslide can be observed in Fig.3-right. Further from these phenomena, coastal subsidence around the basin can be observed on selected areas, as shown in Fig. 3-left.

The topographic phase compensation has been performed with the SRTM DEM, which was acquired in February 2000. The master scene was acquired in December 2009. During this time, the mean Lake Mead water level went down from approximately 370 to 334 meters [8]. As a consequence, areas that used to be under the water in 2000, were new coastal areas in 2009. This phenomenon can be clearly observed in the topographic update estimates. In Fig.4-left green means no topographic update, whereas red-orange-yellow is negative update, i.e. regions that before used to be under the water, as the small island.

Gaps in the estimation results can be observed in Fig.4left. These are due to the burst synchronization and to the PSI requirement that we see the target in all or most of the images. If enforced that a point must be visible in all images, then a gap of 125-150 meter would have no estimates. For this visualization this condition was relaxed to PS visible in at least 59 images.

Figure 4-center shows the average PS time coherence calculated on a $100 \times 100$ grid. Points in black mean that a minimum of 10 PSs was not reached. The coherence is homogeneously distributed, with some local variations which seem to be correlated with topography. Figure 4right shows histograms of PS time coherence for $200 \mathrm{~Hz}$ Doppler Centroid bins, with the local Doppler Centroid of the bin used as colour code. It can be seen that the distribution of coherence is almost the same independently of the Doppler Centroid.

\section{EXPLOITATION OF OVERLAP AREAS}

The ScanSAR and TOPS SAR acquisition modes have overlap between bursts within a beam as well as between beams. ESD exploits these overlaps during coregistration in order to accurately estimate the azimuth co-registration offset. As already proposed in [5], it is possible to exploit the ESD technique with the PSs interferometric phases. One advantage of PSs is that they do not decorrelate (or very slowly) in time and thus they can be exploited even with master and slaves images having a high temporal separation, i.e. when the coherence of distributed scatterers decreases due to natural decorrelation phenomena [11].

The approach we suggest is to exploit the PSs at the overlap areas after PSI detection in order to perform a quality control of the azimuth co-registration accuracy.
As previously mentioned, PS detection was performed on a partition composed of the central areas of the bursts. We will call these detected PS "primary PS", since they have been detected due to their SCR and subpixel position determination has been performed in their mean amplitude map. For the other areas of the bursts, instead of performing again an independent detection, PSs are extracted in the same positions that PSs were detected in one of the neighbouring bursts. This extra PSs will be called "secondary PSs", since they have not been "detected" directly themselves. It follows that each pair of primary-secondary PSs should provide two observations at different squints from the same target. This concept is visualized in Fig.5-left. Depending on the burst combinations, we obtain primary-secondary pairs with Doppler Centroid differences around $2000 \mathrm{~Hz}$ (intra beam), $1500 \mathrm{~Hz}$ (inter beam) and $500 \mathrm{~Hz}$ (inter beam). The locations of the two first groups are depicted in Fig.5-center and right. Note that the first beam, which was acquired with a lower range bandwidth, has been excluded for the analysis of the primary-secondary PSs.

Firstly the nature of this pairs has been analysed. An evaluation of the coherence of primary versus secondary PSs for the $1500 \mathrm{~Hz}$ group is shown in Fig.6-left. The coherence of the secondary PS is mostly lower than that of the primary. The histogram of coherence-loss versus power-loss for primary-secondary PSs shown in Fig.6center indicates that this lower coherence of the secondary PS could be explained by a power loss. This would be an indicator that PSs in this dataset have a spatial extent and directivity, which is actually consistent with the fact that there are almost no manmade structures. By observing these PSs with different Doppler Centroids we observe different parts of the backscattering pattern and thus different backscattering power. Since the primary PSs are detected on the basis of their SCRs, it is reasonable that they have on average a higher power and coherence.

The PSs that we are exploiting in this dataset are possibly mostly stable rocks. This is further supported by the increase of the standard deviation of the PSs residual phase (jointly evaluated for each interferogram) as the time separation from master increases, which is shown in Fig. 6-right. The standard deviation has been evaluated with primary PSs with coherences between 0.7 and 0.9 . This behaviour is an indicator of decorrelation phenomena, which is reasonable given the short wavelength of X-band.

The behaviour of the three primary-secondary groups is surprisingly similar and requires further analysis. A comparison of the deformation estimates for primarysecondary pairs exhibits biases in the order of hundredths of $\mathrm{mm} /$ year deformation and a hundredth of $\mathrm{m}$ residual topography.

Finally, a preliminary evaluation of the applicability of these primary-secondary PS pairs for azimuth coregistration quality control is illustrated in Fig.7. For 

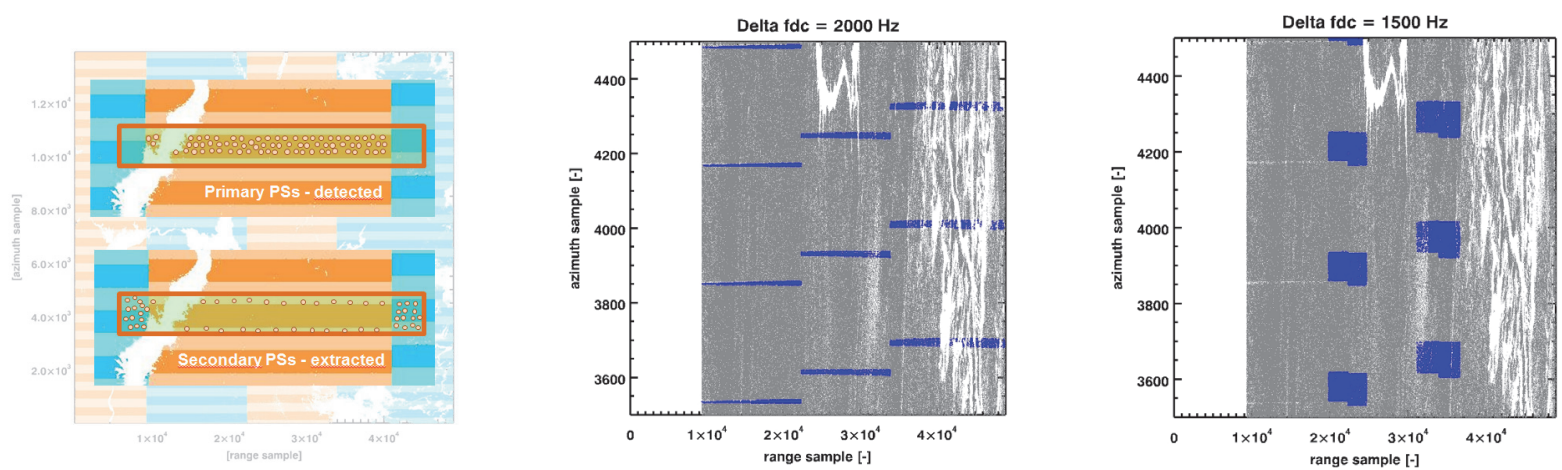

Figure 5. Primary-secondary PSs: (left) primary versus secondary PS; (center) PSs with 2000Hz Doppler Centroid difference; (right) analogous with $\sim 1500 \mathrm{~Hz}$ difference. Selected PSs valid at least for at least 51 interferograms.
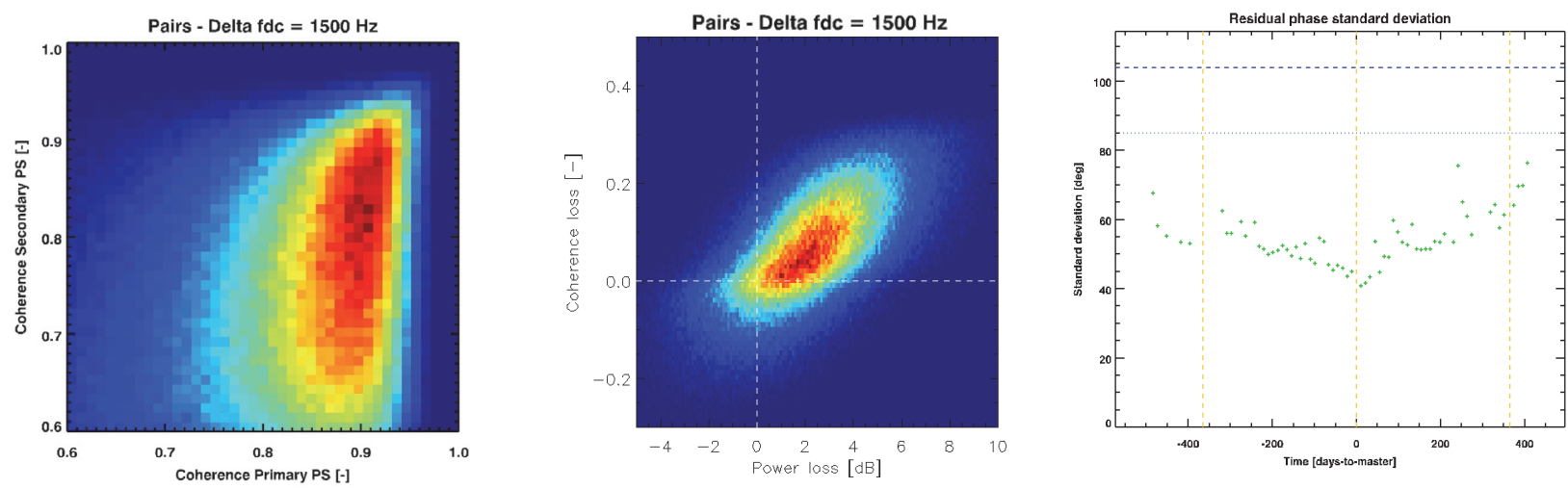

Figure 6. Analysis of primary-secondary PS: (left) 2D histogram of coherences for the 1500Hz Doppler difference group; (center) 2D histogram of coherence loss versus power loss for the $1500 \mathrm{~Hz}$ Doppler difference group (evaluated on pairs where both primary and slave have coherence higher than 0.6); (right) standard deviation of PS residual phases evaluated for each interferogram (evaluate on primary PSs with coherence from 0.7 to 0.9). Selected $P S s$ valid in all interferograms.
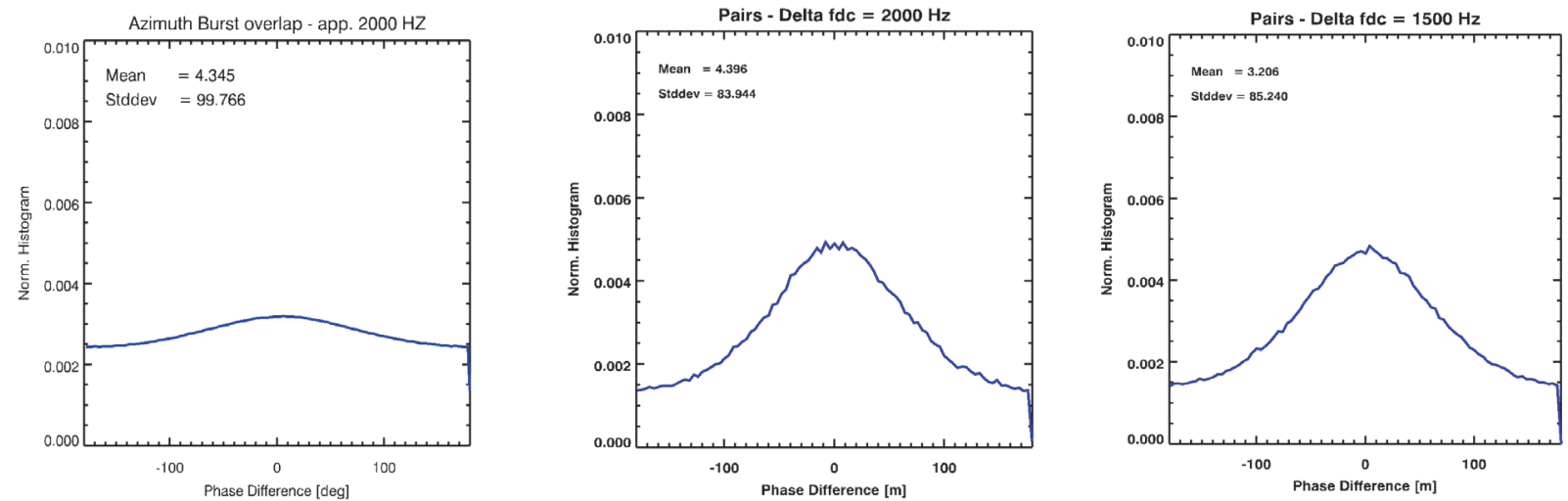

Figure 7. ESD for co-registration quality control-example slave acquired on 19 December 2012 - histograms of single look differential phase: (left) standard ESD in interferogram generation; (center) PS ESD applied to the $2000 \mathrm{~Hz}$ Doppler Centroid difference group; (right) PS ESD applied to the 1500Hz Doppler Centroid difference group. Selected PSs valid at least for at least 51 interferograms.

this purpose, an interferogram with high co-registration error has been selected. On the left side, the histogram of the standard ESD residual phase after interferogram generation (distributed scatterer, intra beam) is depicted. The histogram of the differential PS primary-secondary phases for the $2000 \mathrm{~Hz}$ and $1500 \mathrm{~Hz}$ Doppler difference groups are respectively shown in Fig. 7 -center and right. The phase difference has been calculated so that the difference of Doppler is consistently positive in order to be a direct indicator of residual co-registration offset. 
Initial analysis on the full stack indicates a good consistency between the average differential phases of the three Doppler difference groups and the average differential phase of standard ESD. Nevertheless, for the $2000 \mathrm{~Hz}$ group, mean phase mismatches with respect to standard ESD of maximum value $1.3^{\circ}$ can be observed. These differences could be partially due to the spatial variation of the azimuth residual shift and the different spatial sampling for standard and PS ESD. For the 1500 $\mathrm{Hz}$ and the $500 \mathrm{~Hz}$ some higher mismatches are found, which require further investigation and could be problematic due to the higher phase-to-azimuth-shift conversion factors.

As a consequence, our current concept is to exploit mainly the $2000 \mathrm{~Hz}$ Doppler Centroid difference group, while the other two groups could be used for the verification of the azimuth co-registration ambiguity band. This approach has already been demonstrated for TOPS SAR co-registration purposes using distributed scatterers [12]. The practical evaluation with PS ESD must still be performed.

\section{ACKNOWLEDGEMENTS}

The WAP is developed within the ESA project Terrafirma with the ESRIN/Contract no. 4000109669/13/I-AM.

\section{CONCLUSION}

The extension of DLR's IWAP to support PSI with ScanSAR and TOPS SAR acquisition modes has been presented.

The extension has been demonstrated with a TerraSAR$\mathrm{X}$ ScanSAR data stack in the area surrounding Lake Mead. Subsidence phenomena and a landslide in Lake Mead basins' have been identified. The impact of the water level change on the residual topography with respect to SRTM has been illustrated.

A preliminary evaluation on the behaviour of PSs observed from different bursts and their potential for coregistration quality control has been performed. Further research on urban and TOPS SAR cases should be performed.

\section{REFERENCES}

1. "GMES Terrafirma: Pan European Ground Motion Hazard Information Service," www.terrafirma.eu.com.

2. N. Adam, W. Liebhart, A. Parizzi, F. RodriguezGonzalez and R. Brcic. "Persistent Scatter Interferometry Wide Area Product Methodology and Final Characteristics". Terrafirma Stage 3, DLR-IMF - Remote Sensing Technology Institute, 2012.
3. F. Rodriguez Gonzalez, N. Adam, A. Parizzi, and R. Brcic. "The Integrated Wide Area Processor (IWAP): A Processor for Wide Area Persistent Scatterer Interferometry". ESA Living Planet Symposium, Edinburgh, September 2013.

4. F. de Zan and A. Monti Guarnieri. "TOPSAR: Terrain Observation by Progressive Scans". IEEE Transactions on Geoscience and Remote Sensing, Vol. 44, No. 9, September 2006, pp 2352-2360.

5. P. Prats-Iraola, R. Scheiber, L. Marotti, S. Wollstadt and A. Reigber. "TOPS Interferometry with TerraSAR-X". IEEE Transactions on Geoscience and Remote Sensing, Vol. 50, No. 8, August 2012, pp 3179-3188.

6. N. Yague-Martinez, F. Rodriguez-Gonzalez, U. Balss, H. Breit, T. Fritz. "TerraSAR-X TOPS, ScanSAR and WideScanSAR interferometric processing". EUSAR 2014 - 10th European Conference on Synthetic Aperture Radar, pp. 945948. VDE Verlag. 03-05 June 2014, Berlin, Germany.

7. N. Yague Martinez, R. Brcic, and B, Schättler, "Sentinel-1A Commissioning Phase Technical Assistance for SAR System Verification, Repeat Pass Consistency Analysis". SAR Signal Processing Team, Remote Sensing. Technology Institute, DLR, Sentinel-1 Technical Assistance, Document S1-TR-DLRIMF-0004, 2014.

8. Bureau of reclamation. http://www.usbr.gov.

9. M. Bara; R. Scheiber; A. Broquetas; A. Moreira, "Interferometric SAR signal analysis in the presence of squint". IEEE Transactions on Geoscience and Remote Sensing, vol.38, no.5, pp.2164,2178, Sep 2000

10. Cavalié, O., M.-P. Doin, C. Lasserre, and P. Briole. "Ground motion measurement in the Lake Mead area, Nevada, by differential synthetic aperture radar interferometry time series analysis: Probing the lithosphere rheological structure". J. Geophys. Res., 112, B03403.

11. A. Parizzi, X. Cong, and M. Eineder. "First Results from Multifrequency Interferometry. A comparison of different decorrelation time constants at L, C, and X Band". In: ESA Scientific Publications (SP-677), Seiten 1-5. ESA FRINGE Workshop 2009, Frascati.

12. N. Sakar. "Development of Advanced Coregistration Methods for Sentinel-1 TOPS Mode". Master thesis. Technische Universität München, December 2014. 